\title{
La situación sociolingüística de la lengua creole de San Andrés Isla: el caso de San Luis ${ }^{1}$
}

\section{The sociolinguistic situation of creole language in San Andres Island: San Luis case study}

\author{
Deyanira Sindy Moya Chaves, M.A. \\ Pontificia Universidad Javeriana \\ Bogotá, Colombia \\ d.moya@javeriana.edu.co
}

http://dx.doi.org/10.14483/udistrital.jour.calj.2014.1.a05

Received: 5-Sept-2013 / Accepted: 14-Jan-2014

To cite this article

Moya, D. S. (2014). La situación sociolingüística de la lengua creole de San Andrés Isla: el caso de San Luis. Colombian Applied Linguistics Journal, 16(1). 55-66.

\section{Resumen}

El presente artículo describe la situación lingüística de un sector tradicional de la Isla de San Andrés, Colombia llamado San Luis: los ámbitos de uso de las diferentes lenguas que allí coexisten; las funciones que cumplen y la relación entre lengua e identidad de los habitantes. Esta descripción surge de un estudio sociolingüístico llevado a cabo durante los años 2009 y 2010. Las conclusiones e interpretaciones de los hechos y de la información proporcionada se logran a partir de encuestas, observación participante, entrevistas e historias de vida. Los principales resultados sugieren la vitalidad de la lengua creole en los sectores tradicionales de la Isla, la expansión de ámbitos de uso del español y la posible pérdida del inglés. Como conclusiones se plantea la importancia de implementar una educación bilingüe intercultural que favorezca la protección y mantenimiento de la lengua creole y la comprensión entre pobladores y hablantes de diversas lenguas.

Palabras clave: San Andrés Isla, creole sanandresano, educación bilingüe, bilingüismo, identidad étnica, diglosia.

\begin{abstract}
This paperaims at describing the linguistic situation of a particular sector on San Andres Island, Colombia called San Luis. It illustrates the use of three different languages, their functions and the relationship between language and speakers' identity based on the results of a sociolinguistic study developed in 2009 and 2010 in this local area. Surveys, participant observation, interviews and life stories were used to collect information on how much these languagesareseen as part of the ethnical identity of native inhabitants on the island. Results show that there is a continuous expansion of Spanish and a possible loss of English on the island which leads to a need ofan intercultural bilingual education in order to promote a better understanding among diverse cultural and linguistic groups.
\end{abstract}

Keywords: San Andres Island, Creole, bilingual education, bilingualism, identity, diglossia.

1 Este trabajo surge como trabajo de grado para optar al título de magíster en Lingüística de la Universidad Nacional de Colombia. 


\section{Introducción}

Este artículo constituye una síntesis de mi trabajo de grado para optar al título de magíster de la Universidad Nacional de Colombia desarrollado en 2010 en San Luis, uno de los sectores tradicionales de la isla de San Andrés donde mayoritariamente habita la población raizal?2. El objetivo principal de esta investigación fue identificar la importancia de tres lenguas que se encuentran en contacto en la isla, su vitalidad, ámbitos de uso y su relación con la identidad étnica de los hablantes. La investigación que se reporta aquí es sociolingüística ya que busca dar cuenta de la relación entre lengua e identidad étnica y cultural. Particularmente, esta investigación constituye un estudio de caso de carácter cualitativo de en un barrio isleño tradicional ubicado en la punta suroriente de la isla. Para describir la situación lingüística de este sector se relacionan variables como edad, escolaridad, ámbitos de uso, e historia familiar de las personas que lo habitan y las lenguas que se encuentran en contacto. Se muestra específicamente cuándo se usan las diferentes lenguas allí presentes, se sugiere cómo cada rango de edad plantea dinámicas bilingües particulares, y se vislumbran posibles tendencias con respecto al futuro de las lenguas en la isla.

Este estudio se llevó a cabo por medio de una encuesta sociolingüística, observación participante y relatos de vida, lo que lo caracteriza como un estudio de corte sociolingüístico. Los principales resultados evidenciaron que la mayoría de la población de tercera generación (mayores de 56) en San Luis es bilingüe tardía creole/español con algunas diferencias respecto al aprendizaje de español como segunda lengua (L2) y adquirió el inglés como tercera lengua (L3). El segundo grupo etario, correspondiente a edades entre 41 y 50 , es mayoritariamente bilingüe secuencial creole/español, siendo el creole su primera lengua (L1). También en este grupo etario hay personas monolingües en español dependiendo de su historia familiar y de si han conformado familias interétnicas. El bilingüismo secuencial creole/español se mantiene para los habitantes del sector que oscilan entre 21 y 40 años de edad, y son quienes mantienen un mayor nivel de composición

2 Raizal hace referencia a la comunidad étnica colombiana que ha conservado la lengua creole y que tiene una cultura afro-anglo-antillana propia con raíces africanas, europeas y caribeñas. de familias interétnicas. Finalmente, el creole se mantiene como L1 en la población raizal más joven, y el español como lengua dominante en la escuela y el trabajo. En cuanto al inglés, se evidenció que éste va desapareciendo de generación en generación. Los relatos de vida mostraron que las actitudes frente al creole son aún positivas ya que esta lengua se sigue manteniendo como bandera identitaria raizal. Sin embargo, aún no hay un reconocimiento por parte de los hablantes del creole como lengua distinta al inglés. Es evidente la paulatina pérdida del inglés en la isla y su reducción a espacios religiosos. El español se mantiene como lengua dominante.

Finalmente, la principal contribución de este estudio sociolingüístico consiste en la identificación de tipos de bilingüismo en la población raizal, el reconocimiento de la vitalidad de la lengua creole en el sector y su importancia como elemento identitario de este grupo étnico.

En el siguiente apartado se presentan los fundamentos teóricos que sustentan esta aproximación sociolingüística a San Andrés Isla. Principalmente, se aclaran conceptos sobre bilingüismo fundamentales para comprender la caracterización que se hace de la comunidad de San Luis.

\section{Aspectos teóricos del bilingüismo}

De acuerdo con Ardila y Ramos (2007), el bilingüismo puede ser estudiado y definido de diferentes maneras. Para empezar, el término bilingüismo incluye a la persona que sabe dos lenguas y también al trilingue o multilingue ya que los fenómenos de interferencia, aprendizaje, préstamos, entre otros, se evidencian cuando se trata de dos o más lenguas (Lastra, 2003). Sin embargo, el término bilingüe se usa para referirse a una persona que puede comunicarse usando dos lenguas diferentes. Los conceptos bilingüe y trilingüe se usan para describir situaciones comparables en las cuales dos o tres lenguas están involucradas. Por su parte, una persona que habla más de dos lenguas es llamada multilingüe así como el contexto o sociedad determinada donde coexisten distintas lenguas. Sin embargo, por economía y simplicidad, bilingüismo es el término más 
frecuente para referirse a la habilidad de hablar dos, tres o más lenguas (Ardila E Ramos, 2007), y así fue como se comprendió en este estudio. De acuerdo con Grosdjean (citado en Ardila \& Ramos, 2007) cerca del $50 \%$ de la población mundial es bilingüe y de allí la importancia de trabajar el tema desde la lingüística y la educación. De hecho, algunos autores estiman que el número de bilingües en el mundo es tan alto como el $80 \%$ de la población (Porch $\mathcal{E}$ Berkeley-Wykes, citado en Ardila E Ramos, 2007). Sin embargo, en la teoría sobre el bilingüismo se afirma que ser bilingüe representa hacer parte de un grupo muy heterogéneo, y por tanto un estudio sobre grupos de personas bilingües o de individuos bilingües estará delimitado por las particularidades de los mismos. Así, comprender su heterogeneidad requiere variedad de mediciones y metodologías de investigación adecuadas al grupo o a los individuos que se estudian.

Para empezar, un hablante bilingüe ha adquirido al menos una lengua durante su niñez llamada primera lengua (L1). De acuerdo con De Mejía (2002), este concepto (L1) generlmente se enmarca en el campo del hablante monolingue en vez del bilingüe por lo cual presupone tradicionalmente que una lengua se adquiere antes que otra o de manera separada. Sin embargo, el desarrollo de este concepto ha llevado a que se use de manera amplia para referirse a la primera lengua adquirida, a la lengua dominante, a la lengua materna o a aquella más usada por el hablante. Así, una L1 hace referencia a un contínuo sobre el desarrollo (primera lengua vs. segunda lengua) y contenido (lengua materna vs. otra lengua), al contexto (lengua extranjera vs. segunda lengua), y al medio en el que adquiere (primera/segunda lengua adquirida) (Mejía, 2002.p.46). Así, un hablante bilingüe tiene la habilidad de usar una lengua extra a la que generalmente se le llama segunda lengua (L2). Las personas pueden ser bilingües al adquirir dos lenguas durante la niñez (bilingüismo simultáneo) o al adquirir la segunda lengua en algún momento posterior al aprendizaje de la primera lengua (bilingüismo sucesivo). Ardila y Ramos (2007) afirman que hay diferentes razones, ya sean sociales o personales, por las cuales se llega a ser bilingüe: debido a la inmigración a otro país, porque se ha vivido temporalmente en un ambiente lingüístico diferente, por vivir en áreas de frontera entre países con lenguas distintas, porque los pa- dres hablan lenguas distintas, o porque se vive en una sociedad bilingüe.

Algunas variables se consideran cruciales a la hora de medir el grado de bilingüismo de un grupo de personas o de individuos. Entre ellas se encuentra la competencia lingüística, la edad, la secuencia de adquisición, la manera en que se adquirieron las lenguas, la lengua de escolarización, el contexto de las dos lenguas, los patrones de uso de las dos lenguas, y las actitudes personales y sociales frente a cada lengua (e.g., Albert y Obler, Manuel-Dupont et al., Kilborn, Paradis, Vaid, citados en Ardila E Ramos, 2007). Ardila y Ramos (2007) agregan las diferencias individuales en la habilidad verbal. Sin embargo, estas son sólo variables generales y existe gran variación. La edad, la secuencia y la manera en que se adquieren las lenguas no se relacionan necesariamente con el grado de competencia de cada lengua. La lengua en que se dio la escolarización puede de hecho ser una variable de gran importancia. Las actitudes sociales e individuales hacia las lenguas pueden ser diversas. Incluso, de acuerdo con Ardila y Ramos (2007.), las diferencias individuales en la habilidad para aprender las lenguas no se han estudiado a profundidad. Evidentemente, existen diferencias significativas en la habilidad para aprender y usar no sólo la primera lengua, sino la segunda también (Ardila E Ramos, 2007).

Existen otras distinciones adicionales con respecto a la competencia lingüística que se encuentran en la literatura sobre bilingüismo (e.g., Crystal, Fabbro, Paradis, Romaine, citados en Ardila E Ramos, 2007). Así, se distingue entre bilingüismo balanceado que define una competencia igual de las dos lenguas y describe una competencia similar a la de un nativo en ambas lenguas. Por su parte, el bilingüismo dominante hace referencia a la preferencia y a un mejor manejo en una lengua. La dominancia de las lenguas varía de acuerdo al contexto donde esas lenguas se usan y también a través del tiempo. El bilingüismo pasivo es aquel que define a un individuo que es capaz de entender las dos lenguas pero que se puede expresar sólo en una de ellas. Bilingüismo substractivo refiere el proceso en el que aprender una segunda lengua lleva a la erosión gradual de la competencia en la primera. Por el contrario, el bilingüismo aditivo describe a quienes adicionan una lengua sin ir en detrimento de la 
competencia en la primera lengua. Es importante mencionar que un bilingüe puede ser clasificado simultáneamente en más de una categoría, y que a medida que se avanza en el aprendizaje de la segunda lengua, la competencia en la misma puede incrementarse.

De acuerdo con el tiempo en el que se adquiere una lengua, los bilingües pueden distinguirse entre (e.g., Bialystok \& Hakuta; Birdsong, Genesee, y Nicoladis, DeKeyser, Flege, citados en Ardila y Ramos, 2007) bilingües simultáneos, tempranos y tardíos. Un bilingüe simultáneo es aquel que tiene dos primeras lenguas (dos lenguas nativas o maternas). Niños que han sido expuestos a dos lenguas desde el nacimiento serán bilingües simultáneos. Si la exposición a la segunda lengua ocurre después de la edad de 3 a 5 años, el término bilingüesecuencial será el más apropiado. El bilingüismo temprano define aquellos individuos que adquieren la segunda lengua antes de que la adquisición de la primera se haya terminado. Esto es, antes de los 12 años de edad. Por el contrario, el bilingüismo tardío describe aquellas personas que aprenden la segunda lengua después de que la adquisición de la primera lengua se haya dado. La segunda lengua entonces se aprende a través de la primera.

Por otro lado, una noción central para describir situaciones de lenguas en contacto en San Luis y en cualquier contexto bilingüe es el de diglosia, el cual hace referencia a todas las situaciones en las que las lenguas presentan diferencias de prestigio y de uso (Siguán, 2001. p.191). Esta noción de diglosia evidencia una relación entre el prestigio social de cada una de las lenguas y la situación social de los individuos y de los grupos que las hablan. De acuerdo con Siguán (2001), la diferencia de prestigio de las lenguas y del nivel social de sus hablantes trae como consecuencia las denominaciones de lengua fuerte para aquella más prestigiosa en contraste con la lengua débil para la menos prestigiosa. Incluso los términos lengua mayoritaria y minoritaria se aplican para describir estas desigualdades sociales y de prestigio de las lenguas. Este desequilibrio entre las lenguas en contacto en una situación de diglosia se da como resultado de las funciones que se cumplen en cada lengua y del uso que sus hablantes hacen de las mismas. Esta división de la sociedad y del uso y prestigio de las lenguas tiene una gran incidencia en el bilingüismo de sus individuos y fue evidente en este estudio. Por un lado, los hablantes cuya L1 es la lengua débil se ven obligados a aprender la lengua fuerte como L2, pero no al contrario, ya que generalmente la lengua débil no se enseña, ni las dinámicas en la sociedad empujan a esta sea la situación, lo que también se presenta como parte de los resultados de esta investigación.

Las situaciones de diglosia son, de acuerdo con Siguán (2001), situaciones dinámicas que generan conflicto y tensiones entre los miembros de estas sociedades debido a relaciones de poder que se establecen y que determinan la distribución, división y desigualdad entre grupos y lenguas. Haugen (citado en Siguán, 2001. p.193) plantea el término esquizoglosia para definir esta ruptura social que se da cuando una lengua se impone como única en un espacio donde confluyen varias. La esquizoglosia propone entonces una nueva relación entre las lenguas en contacto: lengua dominante y lengua dominada. Dominación que puede darse de diversas formas ya sea como represión total al uso de la lengua dominada, o como invisibilización de la misma en ciertos espacios creando la sensación de tolerancia pero ejerciendo la exclusión indirectamente (Siguan, 2001 pp. 191), aunque de hecho la lengua dominante no sea necesariamente la que mayor número de hablantes tenga.

Para concluir, estos fenómenos de bilingüismo y contacto de lenguas fueron los que me motivaron a llevar a cabo una investigación sociolingüística enfocada en un pequeño espacio insular bilingüe colombiano donde se hacen evidentes tensiones y situaciones de conflicto causadas por el contacto de tres lenguas.

\section{Perspectivas investigativas sobre el bilingüismo en la isla}

Lo que hoy sabemos sobre la situación lingüística de la isla es relativamente poco y los estudios al respecto parecen estar reducidos a ejercicios investigativos de tipo formativo como trabajos de grado y tesis de maestría. Siendo un contexto en el que se habla una de las dos lenguas creole de Colombia, los avances investigativos sobre ella todavía requieren mayor profundidad. En general, los estudios sobre lenguas creole son relativamente recientes, 
y el interés científico por estas variedades lingüísticas sólo fue esporádico en el pasado (Patiño, 2000. p.104)

Dentro de los estudios más recientes sobre la lengua creole de San Andrés que han tenido una mirada sociolingüística se encuentra la monografía de Connie Davis Pang (2011) que buscaba analizar la lengua criolla sanandresana en su relación con el español y el inglés. La autora también usó como instrumento de recolección de datos una encuesta que tenía el objetivo de identificar las lenguas que habla una muestra de 195 raizales; los ámbitos de uso de esas lenguas y sus actitudes frente a las mismas. Adicionalmente, la estudiante de pregrado usó la observación participante en la comunidad raizal y llevó a cabo entrevistas. Por último, comparó las variantes lingüísticas entre el inglés criollo y el inglés estandarizado. Davis concluye que gran parte de la comunidad raizal habla la lengua nativa y mantiene una lealtad lingüística hacia el creole a pesar de la desigualdad en la que se encuentra en relación con el español. Este ejercicio de investigación formativa confirma resultados obtenidos en estudios previos en cuanto a la importancia del creole para la población raizal. También intenta dar cuenta de fenómenos de interferencia lingüística presentes en la lengua criolla sanandresana. Sin embargo, los resultados se generalizan para toda la isla sin tener en cuenta las diferencias poblacionales propias de este contexto insular. Para comprender la complejidad del Archipiélago considero importante caracterizar la situación lingüística de diferentes sectores poblacionales con el fin de lograr generalizar y mostrar tendencias en el uso de las lenguas y variaciones en las mismas. Mi estudio aporta a esta complejidad ya que describe la situación sociolingüística del sector tradicional de San Luis.

Inge Valencia (2010) por su parte, se preguntó por los elementos que construyen la identidad isleña que van más allá de los que se pueden definir como manifestaciones culturales propias de esta población. Buscaba dar cuenta de esos elementos por recuperar, mantener y proteger; y quería identificar los dispositivos identitarios raizales, isleños, que han permitido un estado de convivencia entre diferentes grupos sociales en el Archipiélago. Valencia concluye que la identidad isleña raizal se ha construido con base en valores relacionados con herencias ingle- sas y africanas; y aquellos que buscan la inclusión y una convivencia intercultural en el Archipiélago. Al respecto, la autora cuestiona esas herencias ya que muestran la particularidad cultural de la población del Archipiélago y legitiman su diferencia, pero sólo con el fin de acceder al reconocimiento de derechos que no toda la población de las islas puede poseer. Por el contrario, Valencia muestra que los valores de convivencia intercultural se establecen desde la composición familiar, el padrinazgo o las relaciones de amistad. Este análisis lleva a la conclusión de que actualmente en el Archipiélago hay un recrudecimiento de las diferencias que ha llevado a confrontaciones entre los distintos grupos sociales y étnicos que allí conviven. Este trabajo buscaba cuestionar la manera en que se configuran los procesos de construcción de nación en nuestro país; y aunque su objetivo no era lingüístico, sus aportes son de gran importancia para comprender las características identitarias de la población raizal, dentro de las cuales la lengua juega un papel importante. En este sentido, mi estudio aporta a la discusión sobre la identidad de la población raizal en relación con su lengua y el uso y mantenimiento de la misma, lo que complementa este trabajo de Valencia.

Unos años más atrás, Silvia Flórez (2006) presenta una mirada sociolingüística a las actitudes lingüísticas de los habitantes del Archipiélago. Con un estudio comparativo, Flórez se interesó por establecer las diferencias existentes entre las islas de San Andrés y Providencia relacionando actitudes lingüísticas y variables de edad, género y etnia. Para tal efecto, realizó una encuesta a 112 personas con edades que oscilaban entre los 14 y los 81 años. Los resultados obtenidos a partir de este estudio comparativo muestran que tanto en San Andrés como en Providencia el español es considerado una lengua importante. El inglés por su parte parece tener una mayor importancia en Providencia que en San Andrés debido principalmente a su carácter de lengua internacional; a su importancia en el turismo y al hecho de que se valora como herramienta importante para el crecimiento personal. La autora también muestra que dentro de las islas, el inglés comparte su carácter formal junto con el español en algunos contextos (Iglesia, Televisión, Radio). Flórez (lbíd.) concluye que en las islas existe una clara conciencia de las ventajas que ofrece el hablar más de una lengua lo que evidencia actitudes favorables 
hacia el multilingüismo. Este estudio aporta considerablemente a la comprensión de la variedad de actitudes lingüísticas que tienen los habitantes del Archipiélago frente a las lenguas que allí se hablan. Sin embargo, no relaciona estas actitudes con la forma en que se aprenden esas lenguas y los tipos de bilingüismo de quienes las aprenden, lo cual hace que mi estudio sea válido para la caracterización de la situación sociolingüística de la isla.

Otra aproximación sociolingüística a la realidad sanadresana enfocada en la alternancia de códigos y prestamos léxicos del creole fue desarrollada por Andrea Urquijo (2005). El objetivo de este estudio era demostrar la presencia de alternancias lingüísticas entre el creole y el español, además de los prestamos léxicos tomados del español por hablantes nativos del creole. Urquijo llevó a cabo entrevistas a raizales de sectores nativos como San Luis y recopiló conversaciones de creole hablantes sobre distintos temas de la cotidianidad que le permitieron analizar los préstamos léxicos más comunes español - creole y las alternancias más recurrentes. La autora concluye que los raizales toman palabras relacionadas con el gobierno, la política, la educación y el campo laboral del español cuando hablan creole, y que a su vez hacen algunos préstamos léxicos del creole hacia el español cuando no recuerdan las palabras en español. Este estudio aporta a la discusión sobre el papel predominante del español en la isla y sobre la importancia de llevar a cabo procesos de normalización del creole. Sin embargo, no presenta ámbitos de uso de estas dos lenguas, como lo hace mi estudio.

Por último, Juliana Andrade (2004) llevó a cabo otro estudio sociolingüístico basado en 660 encuestas. El propósito de este era determinar, por un lado, cuál es la lengua más hablada por los habitantes de la isla, y por otro, identificar los ámbitos de uso y el valor que se le da a las diferentes lenguas que allí se hablan. Los resultados obtenidos por Andrade muestran que en el $68,5 \%$ de los hogares sanandresanos se habla español, mientras que en un $20,3 \%$ de los hogares se habla la lengua creole. En cuanto al valor de las lenguas, Andrade afirma que el inglés es la lengua menos utilizada en la cotidianidad pero es la que más agrada en la isla. El español por su parte no es la lengua que más agrada pero es la preferida para ver televisión y oír la radio. El creole por su parte, es la menos preferida para estas actividades, sin embargo, es la segunda que más gusta. Adicionalmente, Andrade concluye que no es el agrado lo que determina el uso de una lengua en la isla de San Andrés sino el contexto (dónde y con quién se habla). El aporte de este estudio radica en ser una aproximación a un mapa sociolingüístico de San Andrés Isla que muestra tendencias de uso y preferencias lingüísticas por zonas. Sin embargo, no muestra tendencias de aprendizaje de las lenguas que son importantes para completar este mapa. Mi estudio entonces contribuye a esta caracterización proporcionando datos de una zona específica de la isla.

A pesar del auge de estudios sociolingüísticos en la isla, la situación de contacto de lenguas que allí se presenta sigue teniendo una gran importancia ya que aún no se ha logrado comprender totalmente la complejidad de este contexto multilingüe. Parece que describir las lenguas que se encuentran en contacto en la isla y las actitudes lingüísticas de sus hablantes no ha sido suficiente. Los intentos siguen siendo desarrollados por estudiantes que han trabajado de manera aislada y con grandes limitaciones. Aún así, todos demuestran que los hablantes de creole en la isla de San Andrés aún tienen opiniones diversas sobre el estatus de la lengua, sobre su escritura, y sobre su inserción en el contexto escolar. De igual manera, se demuestra que las políticas lingüísticas y educativas locales no han considerado al creole dentro del sistema escolar y que esto ha incidido para que la población raizal siga sintiendo que sus derechos no son respetados.

Esta investigación contribuyea la comprensión de la situación lingüística del Archipiélago ampliando la descripción sobre los tipos de bilingüismo de los habitantes raizales; los ámbitos de uso de las variedades que se encuentran en contacto en un barrio raizal tradicional, y las percepciones que tienen los hablantes sobre el creole.

\section{Metodología}

Fundamentalmente, este estudio sociolingüístico se basa en una encuesta hecha a 44 personas que constituye aproximadamente el $23 \%$ de las personas que habitan los barrios de San Luis ubicados desde Bay hasta Hoffie en ese sector, en donde de acuerdo con el DANE (2005) hay aproximadamente 
187 habitantes y 74 familias. Estas 44 personas se distribuyeron en cuatro grupos etarios distintos. El primero de ellos corresponde a 9 adultos mayores; el segundo grupo a 11 personas con edades entre 41 y 50; el tercer grupo lo conformó un total de 18 personas entre 21 y 40 años de edad; y el último, 6 jóvenes con edades entre 15 y 20 . El objetivo principal de la encuesta era identificar ámbitos de uso de las lenguas, su vitalidad y tipos de bilingüismo de los hablantes. Se escogieron cuatro grupos etarios con el fin de determinar las dinámicas de uso de las diferentes lenguas que allí se hablan, evidenciar cómo cada rango de edad plantea dinámicas bilingües particulares, y vislumbrar posibles tendencias con respecto al futuro de las lenguas en la isla.

Adicionalmente, se recopilaron 25 relatos de vida de líderes comunitarios y personas reconocidas por la comunidad en este pequeño sector de San Luis. El objetivo de estos relatos fue identificar las actitudes lingüísticas de los habitantes del sector, la relación entre lengua e identidad étnica, y las dinámicas de transmisión de las lenguas. Finalmente, la observación participante llevada a cabo durante seis meses en la localidad de la isla permitió aproximarse a la comunidad isleña y comprender la situación de contacto de lenguas que allí se presenta. Se observaron principalmente eventos comunicativos cotidianos en el sector donde se hacía uso de las lenguas en cuestión tal como acontecimientos públicos a los que asistían los hablantes, eventos deportivos, actividades culturales y religiosas, desfiles, entre otros. Se observaba el uso de las lenguas, la alternancia de las mismas, los ámbitos de uso y las actitudes de los hablantes al usarlas. Estas situaciones comunicativas se registraron en un diario de campo. Fundamentalmente, los resultados presentados en este artículo surgen de estos instrumentos.

\section{Resultados}

Los resultados presentados aquí fueron tomados sólo de una parte de la investigación que buscada describir la situación sociolingüística de la lengua creole de San Andrés Isla. Se toman específicamente aquellos que dan cuenta de los tipos de bilingüismo de los hablantes y de los ámbitos de uso de las lenguas que se hablan en un sector específico de este territorio insular. La investigación buscaba en su totalidad caracterizar la comunidad de habla de
San Luis, dar cuenta de las actitudes lingüísticas y la elección de lenguas de los hablantes; identificar la función identitaria de las lenguas y describir las políticas linguísticas en el Archipiélago, así como describir un contexto escolar en la isla donde se privilegia el uso del creole. Los resultados obtenidos en la parte de interés de este capítulo sugieren que independientemente de la lengua de los padres, el contexto tradicional de San Luis favorece el aprendizaje y uso del creole para los hijos de familias interétnicas. De este modo, aunque el padre sea monolingüe en español y les hable en español a sus hijos en el hogar, el barrio y los vecinos raizales favorecen el aprendizaje del creole y por tanto, un bilingüismo temprano, simultáneo y aditivo para estos chicos de familias interétnicas.

Por su parte, de acuerdo a la información recopilada en la encuesta sociolingüística, sólo el $27 \%$ de los hijos de familias raizales han hecho estudios formales de inglés y es posible entonces afirmar que ésta constituye generalmente la L3 de este grupo étnico. Podrían entonces describirse como una población de bilingües simultáneos. Aun así, los resultados también evidencian que es común encontrar en la Isla bilingües pasivos en inglés (logran comunicarse generalmente con hablantes de inglés porque acomodan su habla por propósitos de convergencia lingüística). Esto sugiere que el creole se hace presente en todos los escenarios de sectores tradicionales como San Luis y en el resto de la Isla, debe compartir espacios con el español. El inglés por su parte sigue siendo una lengua de uso cotidiano casi exclusivo del contexto religioso protestante. Los resultados de la encuesta también sugieren que la mayoría de raizales adultos en San Luis, el 53\%, no hablan inglés estándar. Sin embargo, la mayoría de testimonios muestran que para un creole-hablante resulta muy fácil aprender inglés estándar al encontrarse entre angloparlantes (diario de campo entrada de marzo 12 de 2010: charla informal con Hernán) ${ }^{3}$. Esto resulta ser coherente con la cercanía lingüística que existe entre el creole sanandresano (que es de base lexical inglesa) y el inglés estándar (de allí la posibilidad de convergencia entre creolehablantes y anglo-parlantes). Aun así, el uso de esta última lengua en el espacio religioso se hace de manera habitual, aprendida y repetida constantemente,

3 Los nombres de los participantes han sido cambiados con el fin de proteger su identidad. 
lo que no da paso a un uso más espontáneo. Esto nos indica que la lengua dominante de los raizales no es sólo una, sino que depende de su interlocutor y del ámbito de uso. Puede ser el creole en espacios familiares y el español en otros espacios locales. Las familias más tradicionales raizales pueden entonces considerarse como bilingües sucesivos y balanceados creole/español. Por supuesto vemos también la situación de diglosia en este espacio urbano de la isla en la que el creole, el español y el inglés tienen diferentes propósitos, usos y prestigio.

El inglés por su parte, cada vez se aprende menos en San Luis, y en la isla, como L2 o L3. Para la población adolescente actual el inglés es una lengua que se enseña como lengua extranjera en la escuela, o que se aprende si se vive en un país angloparlante fuera de la Isla. Igualmente, aunque se mantenga el inglés en la iglesia,esta función no parece incidir en la población para adquirirla como L2 o L3. Aún así, es interesante resaltar que los jóvenes de San Luis son bilingües sucesivos y que hay variedad en su L1 (creole o español), lo que también muestra la complejidad de la población bilingüe de San Luis y de la Isla.

Todos estos hechos evidencian que para comprender la situación lingüística de San Andrés Isla, se deben tener en cuenta diversas variables que inciden en el uso de una u otra lengua:el interlocutor, los ámbitos de uso, y las funciones de las lenguas. Con respecto a este último factor, el creole cobra gran importancia para crear lazos de amistad y de comunidad étnica; el inglés tiene gran importancia religiosa; y el español cumple funciones principalmente de integración a la sociedad colombiana continental; lo que evidencia de nuevo la situación de diglosia que se vive en este espacio insular.

En cuanto al creole se refiere, la encuesta muestra que los jóvenes lo usan constantemente, pero la mayoría de las personas adultas consideran que ellos no lo hablan debido, principalmente, al hecho de que hay una gran presencia de personas continentales monolingües en español en la isla con quienes los jóvenes raizales estudian, interactúan y establecen relaciones cercanas. Esto indica que la función del español se ha extendido para crear también lazos personales entre raizales y no raizales. Hay así un importante número de familias interétni- cas en la isla y en el sector de San Luis que incide en la percepción de la pérdida de la lengua y de las tradiciones. Además, la población joven siempre quiere salir de la Isla y esto genera una idea por parte de los adultos de pérdida de tradiciones. El gran auge del turismo también ha incidido para que los jóvenes isleños se relacionen más con personas hispanohablantes en español y generen la percepción de los adultos de pérdida de la lengua creole.

Con respecto a la función del español, los resultados de la encuesta sociolinguística en el sector de San Luis evidencian que aunque el creole se mantiene como L1 en la isla, el español se aprende principalmente de manera secuencial como L2. Este aprendizaje secuencial está dado principalmente por la función predominante del español en la esfera escolar, que a su vez incide en la decisión de los padres de familia de enseñar el español en el hogar. Así, los raizales en su mayoría son bilingües tempranos que adquieren la lengua creole como L1 y el español como L2 en el hogar, en la escuela y en la comunidad. Lamentablemente, se evidencia que las funciones que antes ocupaba el creole ahora están siendo desplazadas por el español.

Aún así, los sectores tradiciones de la Isla como San Luis aún constituyen comunidades de habla creole. La encuesta sociolingüística también deja entrever la importancia de la madre en el mantenimiento de la lengua creole. Si la madre se mantiene en la decisión de enseñar creole, lo hace siendo consciente de la importancia de enseñar la lengua a sus hijos como L1. Las redes de solidaridad entre raizales hacen que la lengua se convierta en un lazo social, en un instrumento, en una bandera que se lleva. Es a través de la lengua que se da esa unión del raizal como de la misma clase, del mismo grupo, una unión de identidad: "todo el que habla creole es raizal" (entrada diario de campo enero 7 de 2009). Esto indica que la función principal de la lengua creole es la de determinar una identidad étnica raizal en la isla.

De hecho, la relación entre el creole y la identidad raizal ha sido fundamental para la preservación de la lengua. Esto se puede entender en parte por la fuerte cohesión que existe entre los miembros de una misma familia y la estrecha relación que se da entre la familia y la tenencia de tierra. Los terrenos 
son generalmente repartidos entre los hijos favoreciendo la transmisión de la lengua. Los abuelos y las primeras generaciones serán entonces las portadoras de ese saber lingüístico y los principales responsables de su transmisión y mantenimiento.

De acuerdo con Appel y Muysken (1996), el uso de las lenguas requiere el empleo de una serie de normas concretas y la especialización funcional de las lenguas implicadas. En este contexto bilingüe, el creole cumple funciones de cohesión étnica y de determinación identitaria al mantenerse en hogares raizales, al ser usado con otros raizales y al ser transmisor de tradiciones afrocaribeñas. En relación con esta última función, el inglés, siendo una lengua cercana con el creole sanandresano, también ayuda a mantener estas tradiciones al cumplir funciones religiosas heredadas de ingleses. El inglés también tiene una carga identitaria más histórica que siempre los remonta a sus antepasados y a la importancia de pertenecer a un Gran Caribe angloparlante. Por otro lado, también tiene más prestigio que el creole para muchos raizales. En ocasiones, es preferible reconocerse como angloparlantes que como creole-hablantes. Esto puede también haberse dado por las dinámicas de turismo tan fuertes en la isla, ya que hablar inglés también favorece la posibilidad de acceder al trabajo con turistas extranjeros.

Aún así, la lengua creole es el símbolo por excelencia de la identidad raizal o nativa, siguiendo los términos de Fishman (1995, p.25): "la lengua da cuenta de la paternidad, expresa el patrimonio y sostiene la fenomenología. Un vehículo que lleve un cargamento de tal valor debe ser considerado igualmente valioso, incluso parte del cargamento, tan valioso en sí mismo y por sí mismo". Además, esta lengua siempre será la iniciadora de la interacción entre raizales y a la vez la identificación de quién es un paisano, un amigo, un familiar; en últimas un raizal. Así, la lengua crea una especie de lazo asociativo (Appel y Muysken 1996, p.26) entre los hablantes y habitantes de San Luis y de la Isla.

Fishman (1995) habla de etnicidad teniendo en cuenta tres dimensiones diferentes: la paternidad, el patrimonio y la fenomenología. La primera de ellas está ligada al sentimiento de continuidad, de adquisición de padres y generaciones anteriores. La segunda dimensión está relacionada con el legado de la colectividad, lo que define al grupo como propio y diferente, la música, las tradiciones, modelos que se han seguido, patrones que se han heredado de generaciones anteriores yla última dimensión, por su parte, hace referencia al significado que se atribuye a ese legado que ha sido heredado. Esto es, las actitudes frente a la pertenencia a un grupo étnico.

Lo observado en la Isla sugiere que el creole tiene una relación muy fuerte con la etinicidad y la identidad raizal. Sigue siendo un vehículo que carga un legado y que es considerado propio y único en este territorio colombiano. Esta lengua cumple por tanto una función integradora, de acuerdo con Appel y Muysken (Op. Cit.),ya que puede mantener una función ritual, y otras señales de identidad siempre que tenga alguna función en la vida privada de los individuos. En la Isla, lo más característico y palpable como identidad étnica seguirá siendo la lengua creole ya que así lo sugieren sus hablantes al mantenerla en el hogar y transmitirla a sus hijos y al describirla como la lengua de los raizales. Otros habitantes de San Andrés que no son considerados raizales también asocian la lengua con la condición étnica raizal. Los llamados outsiders (foráneos o continentales), pañas, o shark head 4 aunque reclaman un espacio en esta comunidad y tienen un interés por formar parte de las dinámicas sociales y culturales de la Isla describen la lengua creole como un puente para relacionarse mejor con los raizales.

En conclusión, la lengua creole tiene una carga identitaria aún fuerte en San Andrés para los raizales, a pesar de la presencia del español y del inglés en la isla, ya que es un símbolo de identidad afrocaribeña y los mantiene unidos como grupo étnico. Esta lengua es también una estrategia de convergencia (a usar la misma lengua) entre los miembros de la comunidad raizal y de divergencia (a usar otra lengua) en algunos casos con pañas, como mecanismo de distanciamiento y división en la Isla.

En la mayoría de los casos es posible encontrarse con situaciones de convergencia que se dan entre raizales y no raizales como estrategias de cortesía al hablar español, pero es más factible que se

4 Los nombres asignados a los foráneos han variado con el tiempo y se mencionan en esferas distintas, por lo que no necesariamente son aceptados por todos los habitantes de la isla. 
presenten estas situaciones si el interlocutor habla inglés. Esto evidencia que hablar inglés puede acercar al outsider con el raizal. Igualmente, si el continental habla creole, es posible que logre comprender mejor las dinámicas sociales y culturales raizales. Esta comprensión por supuesto debe ir en dos vías, y esto sólo se logrará a través de una educación intercultural que promueva no sólo el aprendizaje de las diferentes lenguas que se hablan en la isla, sino el entendimiento entre grupos sociales, la promoción de la tolerancia, y del respeto por las diferencias. Es a partir de las lenguas que se puede comprender las dinámicas socioculturales de los habitantes no sólo de San Luis, sino de San Andrés Isla; por tanto, disminuir la diglosia presente en este espacio insular sólo se logrará a través de la educación intercultural. Las diferentes comunidades de habla podrían entenderse mejor si se convirtieran en hablantes bilingües tempranos, simultáneos y balanceados. Esta es una tarea a largo plazo que requiere de cambio de actitudes frente a las diferentes lenguas; una tarea que no es fácil de cumplir sin el apoyo de los líderes, de la comunidad y de los académicos que trabajan en la isla.

\section{Conclusiones}

La situación lingüística de la isla se ha dado por razones históricas que igualmente inciden en la actual percepción de la gente frente a la lengua creole y a las demás lenguas y hablantes. El sector de San Luis sigue siendo un sector tradicionalmente raizal y su aproximación a través de este estudio logra sugerir que también se sigue manteniendo como un sector bilingüe complejo ya que los hablantes tienen L1 diferentes y la adquisición de las L2 es diversa también. Esto comprueba una vez más que cada individuo bilingüe y cada contexto bilingüe es único, variado y complejo. Las encuestas, la observación participante y la recolección de historias de vida demuestran que desde los abuelos hasta los más jóvenes aún se habla creole a pesar de la gran presencia de hispanohablantes en la isla y de la dominancia del español en la vida oficial de la isla. El inglés por su parte parece perder espacios y hablantes, pero no prestigio. Aquellas personas mayores que fueron escolarizadas en inglés están envejeciendo cada vez más y ya dejarán de transmitir esta lengua a sus hijos, nietos y bisnietos. La iglesia jugaba un papel importante en la enseñanza del inglés a través de SundaySchool, y los colegios están apenas en el proceso de incorporar la enseñanza del inglés como L2 en sus currículos. Aún así, los creole-hablantes en ocasiones se consideran angloparlantes (o usuarios de un inglés mal hablado) debido al prestigio que esto carga. Las nuevas dinámicas de promoción del inglés en la isla (a través del turismo y de la enseñanza de esta lengua en la isla) han hecho que incluso se incremente su prestigio y uso. Sería interesante estudiar aspectos relacionados con la competencia comunicativa de quienes se consideran anglo-parlantes en relación con su actual auge en la isla. También se requieren estudios comparativos más recientes entre el inglés hablado en la isla y el creole sanandresano. Es posible que exista la necesidad de legitimar la creencia de muchos raizales de que son una misma lengua, o que constituyen una lengua caribeña. Esto podría otorgarle mayor prestigio al creole y combatir actitudes negativas aún existentes sobre él entre los habitantes de la isla.

Aunque los habitantes del sector y de la isla tienen un gran interés porque sus hijos aprendan inglés y se esté promocionado bastante el uso de esta lengua en la isla por parte del MEN, de instituciones como el SENA, y programas de inmersión en inglés, sus ámbitos son muy reducidos y algunos creole hablantes no se sienten cómodos al usar inglés entre ellos porque no resulta apropiada para propósitos comunicativos de solidaridad y cercanía. Esto hace que el contexto y los hablantes sean contradictorios y tengan visiones encontradas y cambien de opinión constantemente sobre las lenguas; así como también incide en que la situación de diglosia se mantenga y se perpetúe.

El valor que se le da a la lengua creole es muy alto hoy en día. Si antes se hablaba de broken English, de patois, de un inglés mal hablado, hoy se considera creole o inglés caribeño. Las percepciones frente a la lengua son ahora más positivas y más favorables para el uso de la misma en ámbitos más extensos como la escuela. Sin embargo, las contradicciones frente a la relación creole-identidad e inglés-prestigio inciden para que los hablantes perciban el creole y el inglés como dos lenguas autónomas. En ocasiones los hablantes se identifican como hablantes de inglés y no de una lengua creole. 
Por su parte, la escritura de la lengua creole como parte de su estandarización aun no ha sido aceptada y está en construcción. Los hablantes prefieren leer y escribir inglés. Aunque consideran que es importante conservar el creole, no ven necesaria su escritura. Adicionalmente evalúan los intentos de escritura actuales de manera negativa porque estéticamente es muy similar al español y no tanto al inglés. Este proceso tomará tiempo para que sea aceptado. Esto afirma aún más la complejidad de la situación sociolingüística de la isla y el conflicto de conservar lo local y lograr competir a nivel nacional e internacional al mismo tiempo.

Construir currículos bilingües interculturales que promueven no sólo el aprendizaje de las diferentes lenguas que se hablan en la isla, sino el entendimiento entre grupos sociales, la tolerancia, y el respeto por las diferencias parece urgente en la Isla.Las actitudes lingüísticas locales parecen ir en contraposición de lo que muchos hablantes perciben sobre su lengua y sobre el sistema educativo actual. Esta visión incide para que una educación intercultural, significativa y pertinente no sólo para la comunidad raizal, sino continental y musulmana esté lejos de lograrse.

Los resultados de este estudio aportan al conocimiento de la realidad sociolingüística de San Andrés Isla ya que describen los diferentes tipos de bilingües que cohabitan en un sector tradicional de la isla. La variedad descrita en este estudio evidencia diferentes formas de adquisición de las lenguas (como L1, L2 o L3; de manera temprana o tardía; de forma simultánea o sucesiva) y de uso que mantienen un estado de diglosia complejo y difícil de cambiar, ya que está dado por representaciones de las lenguas principalmente construidas sobre hechos históricos que ha marcado a los hablantes. Comprender cómo se aprenden las lenguas que se usan en un contexto específico y sus usos resulta ser información de gran importancia para construir currículos incluyentes que se ajusten a las necesidades de los hablantes. Este estudio de caso en la isla de San Andrés muestra la necesidad de llegar a acuerdos entre los intereses políticos en materia lingüística y aquellos de la comunidad. No sólo los creole hablantes quieren que su lengua tenga un espacio en el contexto escolar, sino que los continen- tales desean que sus hijos aprendan creole e inglés para que sean aceptados como parte de la sociedad isleña. Se entrevé la necesidadde promover una educación bilingüe intercultural en la Isla con el fin de evitar que se sigan generando dificultades en el aprendizaje de niños raizales y distancias culturales entre los diferentes grupos que habitan la isla.

En relación con esta necesidad, actualmente un grupo interdisciplinario, interinstitucional e intercultural trabaja en la definición de una política lingüística participativa para el Archipiélago. Este carácter participativo se ha logrado con el desarrollo de debates internos; de consultas hechas a la comunidad en mesas sectoriales en San Andrés y en Providencia; y con base en investigaciones y fundamentos teóricos. Sanmiguel (2012) afirma que el proceso ha estado lleno de retos y tensiones que afrontar y superar debido a los discursos encontrados con respecto a las diferentes lenguas y al papel que deben jugar en las dinámicas educativas, sociales y culturales del Archipiélago. El debate continúa y en él se han involucrado los medios y la comunidad. Como lo afirma Sanmiguel (lbíd. p.8) "Ese ha sido el mayor logro de este proceso, atravesado por intereses de poder y liderazgo".

Como conclusión se puede afirmar que la investigación sobre el Archipiélago debe continuar y que los trabajos investigativos que se sigan desarrollando sobre la situación lingüística de la isla tienen que involucrar a la comunidad y hacerse con equipos interdisciplinarios. Pequeñas acciones aisladas seguirán demostrando, con todas sus limitaciones, la necesidad de llegar a acuerdos entre las partes, a decidir si se debe normalizar e incluir el creole en el sistema educativo y a reconocer su importancia para la identidad raizal; pero no lograrán iniciar procesos de acción y de planificación lingüística específicos. Cualquier investigación que continúe debe incluir estudios lingüísticos profundos sobre la lengua criolla sanandresana en relación con su herencia africana y de su fonología, morfología y sintaxis; y en cuanto a la influencia del español y del inglés en la misma. Se requieren también estudios de tipo investigación-acción participativa encaminados hacia la construcción de currículos interculturales bilingües incluyentes y pertinentes para los habitantes las islas. 


\section{Referencias}

Andrade, J. (2004) Una aproximación al atlas sociolingüístico de San Andrés [trabajo de grado], Bogotá, Universidad Nacional de Colombia, Carrera de Lingüística.

Appel, R. Y Muysken P. (1996). Bilingüismo y contacto de lenguas. Barcelona, Ariel.

Ardila, A (2012) "Ventajas y desventajas del bilinguismo." en Forma y Función, vol. 25, No 2 julio - diciembre del 2012. Bogotá, Colombia pp.99-114.

Ardila, A. Y Ramos, E. (2007). Speech and Language Disorders in Bilinguals. New York: Nova Science Publishers Inc.

Davis, P. (2011). Análisis sociolingüístico del conflicto entre el español, el inglés y la lengua nativa en San Andrés Isla [trabajo de grado], Pereira, Universidad Tecnológica de Pereira, Carrera de Licenciatura en español y literatura.

De Mejia, A. (2002) Power, Prestige and Bilingualism: International Perspectives on Elite Bilingual Education. Multilingual Matters LTD.
Florez, S (2006, ene-dic.)."A Study of Language Attitudes in Two Creole-Speaking Islands: San Andres and Providence (Colombia)", en Ikala, Revista de lenguaje y cultura, vol. 11, No 17, 2006, pp.119-147.

Fishman, J.A. (1995). Bilingual Education: An International Sociological Perspective. Rowley, MA: Newbury House.

Patiño, C. (2000). Sobre etnolingüística y otros temas. Instituto Caro y Cuervo, Santafé de Bogotá.

San Miguel, R. (2012). "Obstáculos para una política de lenguas en San Andrés”, en UN Periódico, núm. 159, p. 8.

Siguan, M. (2001). Bilingüismo y lenguas en contacto. Madrid. Alianza Ensayo.

Urquijo, A. (2005). Alternancia de códigos y préstamos léxicos del criollo sanandresano [trabajo de grado], Bogotá, Universidad Nacional de Colombia, Carrera de Lingüística.

Valencia, I (2010). "Identidades del Caribe insular colombiano: Otra mirada del caso isleño-raizal". En SC No. 02 - Agosto 2008. Abril, 2010. p. 51-73.

\section{THE AUTHOR}

DEYANIRA SINDY MOYA, M.A. in Linguistics and Bilingualism, and a Bachelor Degree on Philology and Languages (English) from Universidad Nacional de Colombia. She is currently a professor at the Pontificia Universidad Javeriana. 